\title{
Algebraic ramifications of the common extension problem for group-valued measures
}

\author{
by
}

\author{
R. G̈̈bel (Essen) and R. M. S hortt (Middletown, Conn.)
}

\begin{abstract}
Let $G$ be an Abelian group and let $\mu: \mathcal{A} \rightarrow G$ and $\nu: \mathcal{B} \rightarrow G$ be finitely additive measures (charges) defined on fields $\mathcal{A}$ and $\mathcal{B}$ of subsets of a set $X$. It is assumed that $\mu$ and $\nu$ agree on $\mathcal{A} \cap \mathcal{B}$, i.e. they are consistent. The existence of common extensions of $\mu$ and $\nu$ is investigated, and conditions on $\mathcal{A}$ and $\mathcal{B}$ facilitating such extensions are given.
\end{abstract}

0. Introduction. We consider the following problem: Let $G$ be an Abelian group and let $\mu$ and $\nu$ be $G$-valued charges (i.e. finitely additive measures) defined on fields $\mathcal{A}$ and $\mathcal{B}$ of subsets of a set $X$. When does there exist a common extension of $\mu$ and $\nu$ to a charge $\varrho$ defined on $\mathcal{A} \vee \mathcal{B}$, the field generated by $\mathcal{A} \cup \mathcal{B}$ ? Clearly, one must assume at least that the charges $\mu$ and $\nu$ are consistent, i.e. $\mu=\nu$ on $\mathcal{A} \cap \mathcal{B}$. Earlier work of K. M. Rangaswamy and J. D. Reid [11] and K. P. S. Bhaskara Rao and R. M. Shortt [4] has shown that the answer is in the affirmative so long as $G$ is the homomorphic image of a compact group (i.e. is cotorsion). In fact, [4] and [11] demonstrate that this property characterizes the class of cotorsion groups.

In the present article, the focus shifts to include consideration of the fields $\mathcal{A}$ and $\mathcal{B}$. In $\S 2$, an invariant $d$ of the pair $(\mathcal{A}, \mathcal{B})$ is introduced: $d$ is a distance function on the Stone space of $\mathcal{A} \vee \mathcal{B}$. The function $d$ provides information about the geometry of $\mathcal{A}$ and $\mathcal{B}$ and the algebraic structure of the ring of simple functions measurable for $\mathcal{A} \vee \mathcal{B}$. See Lemmas 2.1 and 2.3 together with Theorem 2.4.

In $\S 3$, the case where $\mathcal{A}$ and $\mathcal{B}$ are isomorphic to power set algebras is considered in terms of the common extension of charges (Lemma 3.1), and

1991 Mathematics Subject Classification: 28B10, 20K15, 20K20.

Work of the second author partially supported by the Fulbright Scholar Program and the Graduiertenkolleg at the University of Essen. 
thence is derived a general result (Theorem 3.2) to the effect that whenever the $d$-function is bounded, common extensions of consistent charges always exist, irrespective of the group $G$. This is the case when the fields $\mathcal{A}$ and $\mathcal{B}$ are independent or even only almost independent, so that common extensions always exist for such fields (Theorem 4.2).

Section 5 gives an application of the techniques used in $\S 3$ to the situation where $\mathcal{A}$ and $\mathcal{B}$ are the spectral fields of continuous functions defined on a compact metric space $X$ (Theorem 5.1).

Sections 6 and 7 stress the algebraic aspects of the relevant quotient group of $\mathcal{A} \vee \mathcal{B}$-measurable simple functions and approach the question of characterizing those torsion-free groups that can arise as groups of $\mathcal{A} \vee \mathcal{B}$ measurable simple functions modulo sums of $\mathcal{A}$-measurable and $\mathcal{B}$-measurable functions. It is shown in Theorem 6.1 that every rational group arises in this way; this class of groups is also shown to be closed under the taking of direct sums. For fixed fields $\mathcal{A}$ and $\mathcal{B}$, the question is posed for which groups $G$ consistent $G$-valued charges on $\mathcal{A}$ and $\mathcal{B}$ have a common extension. The notion of general cotorsion theories is applied to solve this problem when both $G$ and the quotient group of simple functions are rational (Theorem 7.1). When the quotient group of $\mathcal{A} \vee \mathcal{B}$-measurable simple functions is a group such as $\mathbb{Z}^{\omega}$, however, it remains an interesting open problem whether a non-cotorsion $G$ exists with the property that all consistent $G$-valued charges on $\mathcal{A}$ and $\mathcal{B}$ have a common extension.

1. Exposition of the problem. All of the groups we consider are Abelian, and we employ the usual additive notation. Let $X$ be a non-empty set and let $\mathcal{A}$ be a field (Boolean algebra) of subsets of $X$. Let $G$ be an Abelian group; a function $\mu: \mathcal{A} \rightarrow G$ is a (G-valued) charge if $\mu\left(A_{1} \cup A_{2}\right)=$ $\mu\left(A_{1}\right)+\mu\left(A_{2}\right)$ whenever $A_{1}$ and $A_{2}$ are disjoint sets in $\mathcal{A}$. If $G=\mathbb{R}$ (or $\mathbb{Z}$ or $\mathbb{Q}$ ) and range $(\mu)=\{0,1\}$, then $\mu$ is said to be a $0-1$ charge. Then $\{A \in \mathcal{A}: \mu(A)=1\}$ is an ultrafilter of $\mathcal{A}$ (considered as a Boolean algebra); furthermore, every ultrafilter of $\mathcal{A}$ arises in this way. Define $S(X, \mathcal{A})$, or $S(\mathcal{A})$ for short, to be the set of all functions $f: X \rightarrow \mathbb{Z}$ such that range $(f)$ is finite and such that $f^{-1}(n) \in \mathcal{A}$ for every $n \in \mathbb{Z}$. These are the $\mathcal{A}$-measurable simple functions on $X$. We see that $S(X, \mathcal{A})$ becomes a ring under pointwise addition and multiplication of functions.

Let $\mu: \mathcal{A} \rightarrow G$ be a $G$-valued charge. Given $A \in \mathcal{A}$, let $I_{A}$ be its indicator function. Then the mapping $I_{A} \rightarrow \mu(A)$ extends uniquely to a group homomorphism from $S(X, \mathcal{A})$ to $G$. The value of this homomorphism at $f \in S(X, \mathcal{A})$ is the integral $\int f d \mu$. Conversely, every group homomorphism from $S(X, \mathcal{A})$ to $G$ can be formed in this way. It is often easier to work with $S(X, \mathcal{A})$ than $\mathcal{A}$ and sometimes easier to work with homomorphisms than with set functions. 
The question of whether a $G$-valued charge can be extended to a charge defined on a larger field of sets has been completely solved by T. Carlson and K. Prikry [5], who proved the following result. Let $\mathcal{P}(X)$ be the power set field of $X$.

1.1. Lemma. Let $\mu: \mathcal{A} \rightarrow G$ be a $G$-valued charge on $(X, \mathcal{A})$. There is a charge $\varrho: \mathcal{P}(X) \rightarrow G$ such that $\varrho(A)=\mu(A)$ for all $A \in \mathcal{A}$.

Indication. The proof relies on the fact, discovered by Nöbeling and Specker, that every ring of the form $S(X, \mathcal{A})$ is, when considered solely as a group, free Abelian and that when $\mathcal{A}$ is a subfield of $\mathcal{B}$, then $S(\mathcal{A})$ is a direct summand in $S(\mathcal{B})$. (A proof of this due to Bergman, as well as general information about such rings, is to be found in $[7 ; \S 97]$.) Thus, one may write $S(\mathcal{P}(X))=S(\mathcal{A}) \oplus F$ for some group $F$. Composing projection onto the factor $S(\mathcal{A})$ with the charge $\mu$ yields the desired extension $\varrho$.

A more elusive problem arises from the attempt to extend two charges simultaneously. Suppose that $\mathcal{A}$ and $\mathcal{B}$ are fields of subsets of a set $X$ and that $\mu: \mathcal{A} \rightarrow G$ and $\nu: \mathcal{B} \rightarrow G$ are charges. We say that $\mu$ and $\nu$ are consistent if $\mu(C)=\nu(C)$ for all $C \in \mathcal{A} \cap \mathcal{B}$. For a given $G$, we ask whether two consistent $G$-valued charges have a common extension, i.e. whether there exists a $G$-valued charge $\varrho$ such that $\varrho(A)=\mu(A)$ for all $A \in \mathcal{A}$ and $\varrho(B)=$ $\nu(B)$ for all $B \in \mathcal{B}$. The charge $\varrho$ is to be defined on $\mathcal{A} \vee \mathcal{B}$, the field generated by $\mathcal{A} \cup \mathcal{B}$. (Then Lemma 1.1 may be employed to obtain a common extension defined on all the subsets of $X$.) Given consistent charges $\mu$ : $\mathcal{A} \rightarrow G$ and $\nu: \mathcal{B} \rightarrow G$, define a homomorphism $\varphi: S(\mathcal{A})+S(\mathcal{B}) \rightarrow G$ by setting

$$
\varphi(f+g)=\int f d \mu+\int g d \nu .
$$

Here, $S(\mathcal{A})+S(\mathcal{B})$ is the subgroup of all sums $f+g$ with $f \in S(\mathcal{A})$ and $g \in S(\mathcal{B})$. One easily checks that $\varphi$ is well defined and that $\mu$ and $\nu$ have a common extension $\varrho: \mathcal{A} \vee \mathcal{B} \rightarrow G$ if and only if $\varphi$ can be extended to a homomorphism $\varphi^{\prime}: S(\mathcal{A} \vee \mathcal{B}) \rightarrow G$. (Then $\varrho(C)=\int I_{C} d \varrho$.) It thus becomes possible to deduce, as in $[4 ; \S 3]$ and $[11 ; 3.6]$, the following result.

1.2. Lemma. Let $\mathcal{A}$ and $\mathcal{B}$ be fields of subsets of a set $X$ and let $G$ be a group. Then every pair of consistent charges $\mu: \mathcal{A} \rightarrow G$ and $\nu: \mathcal{B} \rightarrow G$ has a common extension $\varrho: \mathcal{A} \vee \mathcal{B} \rightarrow G$ if and only if $\operatorname{Ext}(H(\mathcal{A}, \mathcal{B}), G)=0$, where $H(\mathcal{A}, \mathcal{B})=S(\mathcal{A} \vee \mathcal{B}) /[S(\mathcal{A})+S(\mathcal{B})]$.

A group $G$ is said to be a cotorsion group if $\operatorname{Ext}(\mathbb{Q}, G)=0$ or, equivalently, $\operatorname{Ext}(H, G)=0$ for all torsion-free groups $H$. (See [7; §54].) It is not hard to prove that $S(\mathcal{A})+S(\mathcal{B})$ is a pure subgroup of $S(\mathcal{A} \vee \mathcal{B})$, so that $H(\mathcal{A}, \mathcal{B})$ is torsion-free. Also, there exists a pair $(\mathcal{A}, \mathcal{B})$ such that $H(\mathcal{A}, \mathcal{B})$ 
contains $\mathbb{Q}$ as a subgroup. (Later, we show that $H(\mathcal{A}, \mathcal{B}) \cong \mathbb{Q}$ is realizable.) Putting all this together yields $([4 ; 3.2]$ and $[11 ; 3.6])$ the following result. Say that a group $G$ has the common extension property if every pair of consistent $G$-valued charges (on any $\mathcal{A}$ and $\mathcal{B}$ ) admits of a common extension.

1.3. TheOREM. A group $G$ has the common extension property if and only if it is a cotorsion group.

This class of groups contains all divisible groups such as $\mathbb{R}$ and $\mathbb{Q}$ as well as all compact Hausdorff topological groups (compare the extension theorem in [1]). The group $\mathbb{Z}$ is not cotorsion, however.

The problem of characterizing the class of groups that always allow extensions is thus solved. Shifting focus to the fields $\mathcal{A}$ and $\mathcal{B}$, we may ask what properties of the pair $(\mathcal{A}, \mathcal{B})$ facilitate common extensions. For example, it was proved in [3; Theorem 4.5] that common extensions always exist when $\mathcal{A}$ and $\mathcal{B}$ are independent fields. This result will be generalized in $\S 4$. We close this section with an observation.

1.4. Lemma. Let $\mathcal{A}$ and $\mathcal{B}$ be fields of subsets of a set $X$. Every pair of consistent charges $\mu$ and $\nu$ on $\mathcal{A}$ and $\mathcal{B}$ (with values in a free group $G$ of rank $2^{|X|}$ ) have a common $G$-valued extension (we quantify here over all possible choices of $G$ ) if and only if $S(\mathcal{A})+S(\mathcal{B})$ is a summand in $S(\mathcal{A} \vee \mathcal{B})$, i.e. $H(\mathcal{A}, \mathcal{B})$ is free.

P r o o f. Suppose that $S(\mathcal{A} \vee \mathcal{B})=[S(\mathcal{A})+S(\mathcal{B})] \oplus F$ for some subgroup $F \subseteq S(\mathcal{A} \vee \mathcal{B})$ and let $\mu$ and $\nu$ be consistent $G$-valued charges. Let $\varphi$ : $S(\mathcal{A})+S(\mathcal{B}) \rightarrow G$ be the homomorphism $\varphi(f+g)=\int f d \mu+\int g d \nu$ described above. Define $\varrho: \mathcal{A} \vee \mathcal{B} \rightarrow G$ by putting $\varrho=\varphi \circ \pi$, where $\pi: S(\mathcal{A} \vee \mathcal{B}) \rightarrow$ $S(\mathcal{A})+S(\mathcal{B})$ is the projection with kernel $F$. We see that $\varrho$ is a $G$-valued common extension of $\mu$ and $\nu$.

Now suppose that $\mathcal{A}$ and $\mathcal{B}$ are such that any pair of consistent charges $\mu: \mathcal{A} \rightarrow G$ and $\nu: \mathcal{B} \rightarrow G$ have a common extension. Let $G$ be the free group $S(\mathcal{A})+S(\mathcal{B})$ and $\mu: \mathcal{A} \rightarrow G$ and $\nu: \mathcal{B} \rightarrow G$ the charges defined by $\mu(A)=I_{A}$ and $\nu(B)=I_{B}$. Then $\mu$ and $\nu$ are consistent, and $\varphi: S(\mathcal{A})+S(\mathcal{B}) \rightarrow G$ is the identity mapping. Since $\mu$ and $\nu$ have a common extension, $\varphi$ extends to a homomorphism $\varphi^{\prime}: S(\mathcal{A} \vee \mathcal{B}) \rightarrow G$. Putting $F=\operatorname{ker}\left(\varphi^{\prime}\right)$ yields $S(\mathcal{A} \vee \mathcal{B})=[S(\mathcal{A})+S(\mathcal{B})] \oplus F$, as desired.

2. The geometry of $\mathcal{A}$ and $\mathcal{B}$. Let $\mathcal{A}$ and $\mathcal{B}$ be fields of subsets of a non-empty set $X$ and let $n \geq 2$ be an integer. A cycle of length $n$ for the pair $(\mathcal{A}, \mathcal{B})$ is a pair of partitions $X=A_{0} \cup \ldots \cup A_{n-1}=B_{0} \cup \ldots \cup B_{n-1}$ of 
$X$ into non-empty sets $A_{i} \in \mathcal{A}$ and $B_{i} \in \mathcal{B}$ such that

$$
A_{i} \cap B_{j} \text { is } \begin{cases}\text { non-empty } & \text { if } i \equiv j(\bmod n) \text { or } i \equiv j+1(\bmod n), \\ \text { empty } & \text { otherwise. }\end{cases}
$$

Call a cycle non-trivial if $I_{A_{i} \cap B_{j}} \notin S(\mathcal{A})+S(\mathcal{B})$ for each pair $(i, j)$ such that $i \equiv j(\bmod n)$ or $i \equiv j+1(\bmod n)$. It is not hard to check that such a cycle is non-trivial if and ony if $I_{A_{i} \cap B_{j}} \notin S(\mathcal{A})+S(\mathcal{B})$ for some pair $(i, j)$ such that $i \equiv j(\bmod n)$ or $i \equiv j+1(\bmod n)$.

Once again, let $\mathcal{A}$ and $\mathcal{B}$ be fields of subsets of a set $X$. Let $\operatorname{St}(\mathcal{A}), \operatorname{St}(\mathcal{B})$ and $\operatorname{St}(\mathcal{A} \vee \mathcal{B})$ be the Stone representation spaces of the Boolean algebras $\mathcal{A}$, $\mathcal{B}$ and $\mathcal{A} \vee \mathcal{B}$ respectively. We define a function $\Phi: \operatorname{St}(\mathcal{A} \vee \mathcal{B}) \rightarrow \operatorname{St}(\mathcal{A}) \times \operatorname{St}(\mathcal{B})$ by putting $\Phi(u)=\left(u_{1}, u_{2}\right)$, where $u_{1}$ and $u_{2}$ are the restrictions of the $0-1$ charge (ultrafilter) $u$ to $\mathcal{A}$ and $\mathcal{B}$, respectively. Clearly, $\Phi$ is a continuous function. For $A \in \mathcal{A}$ and $B \in \mathcal{B}$, we see that $u(A \cap B)=0$ if and only if either $u(A)=0$ or $u(B)=0$. It follows that the mapping $\Phi$ is one-one and therefore a homeomorphism of $\operatorname{St}(\mathcal{A} \vee \mathcal{B})$ onto $R=R(\mathcal{A}, \mathcal{B})=\operatorname{range}(\Phi)$. Since any 0-1 charge on $\mathcal{A}$ (or on $\mathcal{B}$ ) can be extended to a 0-1 charge on $\mathcal{A} \vee \mathcal{B}$, we see that $\pi_{1}(R)=\operatorname{St}(\mathcal{A})$ and $\pi_{2}(R)=\operatorname{St}(\mathcal{B})$, where $\pi_{1}$ and $\pi_{2}$ are the coordinate projections from $\operatorname{St}(\mathcal{A}) \times \operatorname{St}(\mathcal{B})$.

Given points $p$ and $p^{\prime}$ in $R=R(\mathcal{A}, \mathcal{B})$, a sequence $p_{0}, p_{1}, \ldots, p_{n}$ in $R$ is a $p$-chain of length $n$ from $p$ to $p^{\prime}$ if $p_{0}=p, p_{n}=p^{\prime}$, and for each $i=0,1, \ldots, n-1$, either $\pi_{1}\left(p_{i}\right)=\pi_{1}\left(p_{i+1}\right)$ or $\pi_{2}\left(p_{i}\right)=\pi_{2}\left(p_{i+1}\right)$. We define $d: R \times R \rightarrow\{0,1, \ldots, \infty\}$ by putting $d\left(p, p^{\prime}\right)=0$ in case $p=p^{\prime}$ and otherwise

$$
d\left(p, p^{\prime}\right)=\inf \left\{n \text { : there is a } p \text {-chain of length } n \text { from } p \text { to } p^{\prime}\right\} .
$$

The function $d$ is a distance, i.e. it is symmetric and satisfies the triangle inequality. Note that $d\left(p, p^{\prime}\right)=\infty$ is possible.

2.1. Lemma. Let $\mathcal{A}$ and $\mathcal{B}$ be fields of subsets of a set $X$ and suppose that $\mathcal{A} \cap \mathcal{B}=\{\emptyset, X\}$. For a fixed positive integer $n$, suppose that $d\left(p, p^{\prime}\right)<2 n$ for all $p, p^{\prime} \in R(\mathcal{A}, \mathcal{B})$. Then all cycles for the pair $(\mathcal{A}, \mathcal{B})$ have length $<2 n$.

Proof. Suppose there exists a cycle of length $\geq 2 n$. It is not hard to see that there is then a cycle of length exactly $2 n$, which we write as $A_{0}, A_{1}, \ldots, A_{2 n-1}, B_{0}, B_{1}, \ldots, B_{2 n-1}$, where the $A_{i}$ and $B_{i}$ are clopen subsets of $\operatorname{St}(\mathcal{A})$ and $\operatorname{St}(\mathcal{B})$, respectively. Choose $p \in A_{0} \cap B_{0}$ and $p^{\prime} \in A_{n} \cap B_{n}$. We assert that $d\left(p, p^{\prime}\right) \geq 2 n$, establishing the lemma.

To see this, suppose that $p=p_{0}, p_{1}, \ldots, p_{N}=p^{\prime}$ is a $p$-chain of minimal length $N=d\left(p, p^{\prime}\right)$ joining $p$ to $p^{\prime}$. But then we see that $p_{i} \in A_{j} \cap B_{k}$, where either $j+k \equiv i(\bmod 2 n)$ or $j+k \equiv-i(\bmod 2 n)$. (In traversing this minimal $p$-chain, each step from $p_{i}$ to $p_{i+1}$ must shift positions from one "box" to the next in the following diagram.) 


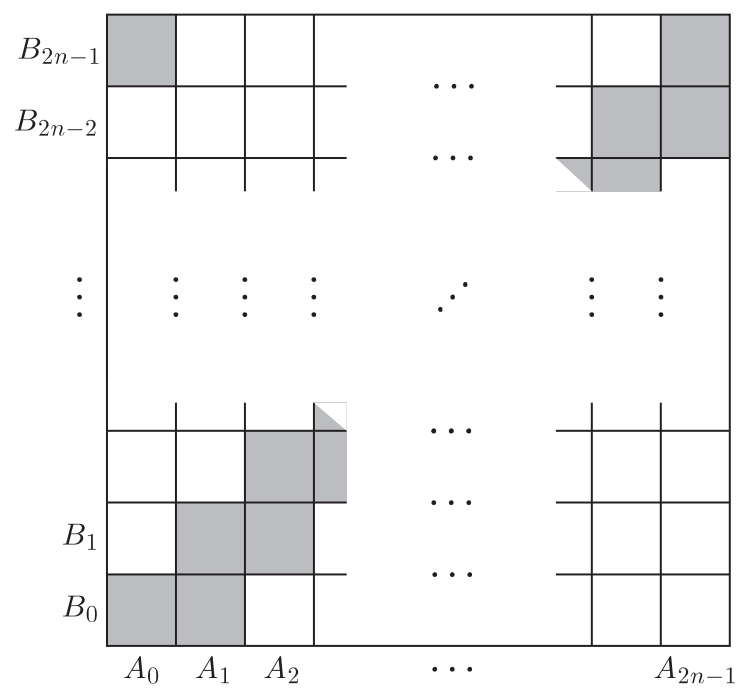

Since $p_{N}=p^{\prime} \in A_{n} \cap B_{n}$, it must be that $N=2 n$, as desired.

2.2. QUESTION. Is the converse of Lemma 2.1 true?

It was proved in $[4 ; \S 2]$ that there exist fields $\mathcal{A}$ and $\mathcal{B}$ on a set $X$ such that $H(\mathcal{A}, \mathcal{B})=S(\mathcal{A} \vee \mathcal{B}) /[S(\mathcal{A})+S(\mathcal{B})]$ contains elements (cosets) that are divisible by every positive integer: $H(\mathcal{A}, \mathcal{B})$ in this case contains $\mathbb{Q}$ as a subgroup. There is a close connection between divisibility of elements of $H(\mathcal{A}, \mathcal{B})$ and the existence of cycles for the pair $(\mathcal{A}, \mathcal{B})$, as explored in the following set of results.

2.3. Lemma. Let $\mathcal{A}$ and $\mathcal{B}$ be fields of subsets of a set $X$ and let $n \geq 2$ be an integer. The following conditions are equivalent:

(i) There is an indicator function $f \in S(\mathcal{A} \vee \mathcal{B})$ representing a non-zero coset $f+[S(\mathcal{A})+S(\mathcal{B})]$ of $H(\mathcal{A}, \mathcal{B})$ that is divisible by $n$ in the group $H(\mathcal{A}, \mathcal{B})$.

(ii) There is a non-trivial cycle of length $n$ for the pair $(\mathcal{A}, \mathcal{B})$.

Pro of. (i) $\Rightarrow$ (ii). If $f+[S(\mathcal{A})+S(\mathcal{B})]$ is divisible by $n$, then we may write $f=n l+g+h$ for some functions $l \in S(\mathcal{A} \vee \mathcal{B}), g \in S(\mathcal{A})$, and $h \in S(\mathcal{B})$. We put, for $i=0,1, \ldots, n-1$,

$$
A_{i}=\{x \in X: g(x) \equiv i(\bmod n)\}, \quad B_{i}=\{x \in X: h(x) \equiv-i(\bmod n)\}
$$

Then $A_{i} \in \mathcal{A}$ and $B_{i} \in \mathcal{B}$ for each $i$, and $A_{0}, \ldots, A_{n-1}$ and $B_{0}, \ldots, B_{n-1}$ are partitions of $X$. We have $f(x) \equiv g(x)+h(x)(\bmod n)$. Since $f$ takes only the values 0 and 1 , it follows that $A_{i} \cap B_{j}=\emptyset$ when $j$ is not congruent to $i$ or $i-1$ modulo $n$. Let $l_{0}$ be the indicator function of the set $A_{0} \cap B_{n-1}$ 
and define

$$
g_{0}=\sum_{i=0}^{n-1} i I_{A_{j}}, \quad h_{0}=\sum_{i=0}^{n-1}-i I_{B_{j}} .
$$

Then $f=n l_{0}+g_{0}+h_{0}$. Since $f \in S(\mathcal{A})+S(\mathcal{B})$, it follows that $l_{0} \in$ $S(\mathcal{A})+S(\mathcal{B})$. Now suppose that the indicator of $A_{0} \cap B_{0}$ is in $S(\mathcal{A})+S(\mathcal{B})$. Then $l_{0} \in S(\mathcal{A})+S(\mathcal{B})$, a contradiction. We now see that $A_{0}, \ldots, A_{n-1}$, $B_{0}, \ldots, B_{n-1}$ is a non-trivial cycle of length $n$.

(ii) $\Rightarrow$ (i). Let $A_{0}, \ldots, A_{n-1}, B_{0}, \ldots, B_{n-1}$ be a non-trivial cycle of length $n$ for the pair $(\mathcal{A}, \mathcal{B})$. We define

$$
\begin{gathered}
f=I_{A_{0} \cap B_{n-1}}+\sum_{i=0}^{n-1} I_{A_{i} \cap B_{i-1}}, \quad l=I_{A_{0} \cap B_{n-1}}, \\
g=\sum_{i=0}^{n-1} i I_{A_{i}}, \quad h=\sum_{i=0}^{n-1}-i I_{B_{i}} .
\end{gathered}
$$

Then $f=n l+g+h$, where $g \in S(\mathcal{A}), h \in S(\mathcal{B})$ and $l \in S(\mathcal{A} \vee \mathcal{B})$. Since the cycle is non-trivial, it follows that $l \notin S(\mathcal{A})+S(\mathcal{B})$. Moreover, since $S(\mathcal{A})+S(\mathcal{B})$ is a pure subgroup, $f$ is the desired indicator with $f \notin$ $S(\mathcal{A})+S(\mathcal{B})$.

2.4. Theorem. Let $\mathcal{A}$ and $\mathcal{B}$ be fields of subsets of a set $X$. Suppose that there is some positive integer $M$ such that every non-trivial cycle for $(\mathcal{A}, \mathcal{B})$ is of length less than $M$. Then for each non-trivial coset $f+S(\mathcal{A})+S(\mathcal{B})$ in $H(\mathcal{A}, \mathcal{B})$, there is a maximal $m$ for which $f+S(\mathcal{A})+S(\mathcal{B})=m f^{\prime}+S(\mathcal{A})+$ $S(\mathcal{B})$ for some $f^{\prime} \in S(\mathcal{A} \vee \mathcal{B})$.

Proof. We note first that when $f$ is an indicator function, the theorem follows directly from the preceding lemma. For a general $f$, we suppose the contrary, i.e. that $f+S(\mathcal{A})+S(\mathcal{B})$ may be divided by arbitrarily large integers. Since constant functions belong to $S(\mathcal{A})+S(\mathcal{B})$, it involves no loss of generality to suppose that $f \geq 0$. Choose now some $n \geq \max \{f(x): x \in X\}$ such that $f=n l+g+h$ for some $l \in S(\mathcal{A} \vee \mathcal{B}), g \in S(\mathcal{A})$ and $h \in S(\mathcal{B})$. We write $g=n g_{0}+g_{1}$ and $h=n h_{0}+h_{1}$, where $g_{i} \in S(\mathcal{A})$ and $h_{i} \in S(\mathcal{B})$ are such that $0 \leq g_{1} \leq n-1$ and $-n+1 \leq h_{1} \leq 0$. Put $l_{0}=l+g_{0}+h_{0}$, so that $f=n l_{0}+g_{1}+h_{1}$. We now see that $l_{0}$ must be an indicator function: if $l_{0}(x) \geq 2$, then $f(x) \geq 2 n+g_{1}(x)+h_{1}(x) \geq 2 n-n+1=n+1$, a contradiction; if $l_{0}(x) \leq-1$, then $f(x) \leq-n+g_{1}(x)+h_{1}(x) \leq-n+n-1=-1$, another contradiction. The first sentence of the proof, applied to $l_{0}$, together with purity of the subgroup $S(\mathcal{A})+S(\mathcal{B})$, shows that $f+S(\mathcal{A})+S(\mathcal{B})$ cannot be divided by arbitrarily large integers, a contradiction.

2.5. Question. Is the converse of Theorem 2.4 true? In particular, if $H(\mathcal{A}, \mathcal{B})$ is free, then are the non-trivial cycles of $(\mathcal{A}, \mathcal{B})$ of bounded length? 
3. Positive results for general fields. We begin this section with a result regarding a case where $\mathcal{A}$ and $\mathcal{B}$ are isomorphic to power set algebras. It and its method of proof shall find application later.

3.1. Power Set lemma. Let $X_{1}$ and $X_{2}$ be non-empty sets and let $X$ be a non-empty subset of the product $X_{1} \times X_{2}$. Assume that the projections of $X$ are all of $X_{1}$ and $X_{2}$. Define $\mathcal{A}=\left\{\left(A \times X_{2}\right) \cap X: A \subseteq X_{1}\right\}$ and $\mathcal{B}=\left\{\left(X_{1} \times B\right) \cap X: B \subseteq X_{2}\right\}$. Suppose that $\mathcal{A} \cap \mathcal{B}=\{\emptyset, X\}$. Then the following are equivalent:

(i) There is an integer $M$ such that every cycle for $(\mathcal{A}, \mathcal{B})$ is of length less than $M$.

(ii) For any group $G$, any two consistent $G$-valued charges on $\mathcal{A}$ and $\mathcal{B}$ have a common extension to a $G$-valued charge on $\mathcal{A} \vee \mathcal{B}$, i.e. the group $H(\mathcal{A}, \mathcal{B})$ is free.

Proof. (i) $\Rightarrow$ (ii). Let $A_{0}$ be an arbitrary singleton subset of $X_{1}$. Put $R_{0}=\left\{\left(x_{1}, x_{2}\right) \in X: x_{1} \in A_{0}\right\}$ and inductively define

$$
R_{i}= \begin{cases}\left\{\left(x_{1}, x_{2}\right) \in X: \exists\left(y_{1}, y_{2}\right) \in R_{i-1} \text { with } x_{2}=y_{2}\right\} & \text { if } i \text { is odd, } \\ \left\{\left(x_{1}, x_{2}\right) \in X: \exists\left(y_{1}, y_{2}\right) \in R_{i-1} \text { with } x_{1}=y_{1}\right\} & \text { if } i \text { is even. }\end{cases}
$$

Set $T_{0}=R_{0}$ and $T_{i+1}=R_{i+1}-R_{i}$; for $i=0,1, \ldots$, put $A_{i}=\pi_{1}\left(T_{2 i-1}\right)$ for $i>0$ and put $B_{i}=\pi_{2}\left(T_{2 i}\right)$. (The $\pi_{i}$ are projections to the factors $X_{i}$.) We see that $A_{i} \times B_{j} \cap X \neq \emptyset$ is possible only if $i=j$ or $i=j+1$. We assert that $A_{i}=\emptyset$ for $i \geq M$ : otherwise, put $C=X_{1}-\left(A_{0} \cup \ldots \cup A_{M-1}\right)$ and $D=X_{2}-\left(B_{0} \cup \ldots \cup B_{M-1}\right)$ and note that $A_{0} \cup C, A_{1}, \ldots, A_{M-1}, B_{0} \cup D$, $B_{1}, \ldots, B_{M-1}$ would be a cycle for $(\mathcal{A}, \mathcal{B})$ of length $M$. Likewise, $B_{i}=\emptyset$ for $i \geq M$. Suppose that $N$ is the largest integer such that $B_{N}$ is non-empty. For each $2 i \leq N$, let $G_{2 i} \subseteq T_{2 i}$ be the graph of a function $x=g_{2 i}(y)$ from $B_{i}$ to $A_{i}$; also, for $2 i+1 \leq N$, let $G_{2 i+1} \subseteq T_{2 i+1}$ be the graph of a function $y=g_{2 i+1}(x)$ from $A_{i+1}$ to $B_{i}$.

Suppose now that $\mu$ and $\nu$ are consistent $G$-valued charges on $\mathcal{A}$ and $\mathcal{B}$, respectively. We can view $\mu$ and $\nu$ as charges defined on the power sets $\mathcal{P}\left(X_{1}\right)$ and $\mathcal{P}\left(X_{2}\right)$. Define charges $\varrho_{i}$ on $\mathcal{P}(X)$ by descending induction, putting $\varrho_{2 N}(S)=\nu\left(\pi_{2}\left(G_{2 N} \cap S\right)\right)$ and defining

$$
\varrho_{2 i-1}(S)=\mu\left(\pi_{1}\left(G_{2 i-1} \cap S\right)\right)-\varrho_{2 i}\left(\pi_{1}\left(G_{2 i-1} \cap S\right) \times X_{2}\right)
$$

and

$$
\varrho_{2 i}(S)=\nu\left(\pi_{2}\left(G_{2 i} \cap S\right)\right)-\varrho_{2 i+1}\left(X_{1} \times \pi_{2}\left(G_{2 i} \cap S\right)\right) .
$$

It is a small matter to verify that the $G$-valued charge $\varrho=\varrho_{0}+\varrho_{1}+\ldots+\varrho_{2 N}$ is a common extension of $\mu$ and $\nu$.

(ii) $\Rightarrow$ (i). We suppose that (i) fails and (ii) holds. Repeat the process used in the first half of the proof to construct the sets $A_{0}, A_{1}, \ldots$ and $B_{0}, B_{1}, \ldots$ 
Now put $Y_{1}=A_{0} \cup A_{1} \cup \ldots$ and $Y_{2}=B_{0} \cup B_{1} \cup \ldots$; then $\left(Y_{1} \times X_{2}\right) \cap X=$ $\left(X_{1} \times Y_{2}\right) \cap X$ is a non-empty set in $\mathcal{A} \cap \mathcal{B}$ and therefore is all of $X$.

We assert that there is some $M$ such that $A_{i}=B_{i}=\emptyset$ for $i \geq M$. If not, define two fields of subsets of $\mathbb{N}$ as follows: $\mathcal{C}$ [resp. $\mathcal{D}$ ] is the field of all subsets of $\mathbb{N}$ that are unions of the sets $\{0\},\{1,2\}, \ldots,\{2 i-1,2 i\}, \ldots$ [resp. the sets $\{0,1\},\{2,3\}, \ldots,\{2 i, 2 i+1\}, \ldots]$. It was shown in $[4 ; \S 2]$ that there are consistent $\mathbb{Z}$-valued charges $\mu$ and $\nu$ on $\mathcal{C}$ and $\mathcal{D}$ with no common extension to $\mathcal{P}(\mathbb{N})$ or, equivalently, to $\mathcal{C} \vee \mathcal{D}$. Define measurable functions $f: X \rightarrow \mathbb{N}$ by putting $f(x)=n$ for $x \in T_{n}$. Define $\mathbb{Z}$-valued set functions $\mu^{\prime}$ and $\nu^{\prime}$ by $\mu^{\prime}\left(f^{-1}(C)\right)=\mu(C)$ when $C \in \mathcal{C}$ and $\nu^{\prime}\left(f^{-1}(D)\right)=\nu(D)$ when $D \in \mathcal{D}$. These are charges on the fields $f^{-1}(\mathcal{C}) \subseteq \mathcal{A}$ and $f^{-1}(\mathcal{D}) \subseteq \mathcal{B}$; extend these (Lemma 1.1) to consistent charges $\mu^{\prime \prime}$ and $\nu^{\prime \prime}$ on $\mathcal{A}$ and $\mathcal{B}$. (Since $\mathcal{A} \cap \mathcal{B}$ is trivial, consistency is almost automatic.) If $\varrho$ were a common extension of these, the $\varrho^{\prime}=\varrho \circ f^{-1}$ would yield a common extension of $\mu$ and $\nu$, a contradiction. Thus, it must be that some $M$ exists with $A_{i}=B_{i}=\emptyset$ for $i \geq M$.

It is not hard to check, much as in the proof of Lemma 2.1, that $M+1$ is an upper bound on the length of cycles for $(\mathcal{A}, \mathcal{B})$.

This lemma facilitates the proof of a result for general fields involving boundedness of the distance function $d$.

3.2. Theorem. Let $\mathcal{A}$ and $\mathcal{B}$ be fields of subsets of a set $X$ and suppose that the distance function $d$ is bounded. Then for any group $G$, any two consistent $G$-valued charges $\mu$ and $\nu$ on $\mathcal{A}$ and $\mathcal{B}$ have a common extension to a $G$-valued charge on $\mathcal{A} \vee \mathcal{B}$, i.e. the group $H(\mathcal{A}, \mathcal{B})$ is free.

P r o o f. We consider again $R(\mathcal{A}, \mathcal{B}) \subseteq \operatorname{St}(\mathcal{A}) \times \operatorname{St}(\mathcal{B})$. Consider also the power set algebras $\mathcal{A}_{0}=\mathcal{P}(\operatorname{St}(\mathcal{A}))$ and $\mathcal{B}_{0}=\mathcal{P}(\operatorname{St}(\mathcal{B}))$. Use Lemma 1.1 to extend $\mu$ and $\nu$ to charges $\mu_{0}$ and $\nu_{0}$ on $\mathcal{A}_{0}$ and $\mathcal{B}_{0}$. We mimic the constructions in the proof of the preceding "power set lemma" treating $R(\mathcal{A}, \mathcal{B})$ as $X$, and $\operatorname{St}(\mathcal{A})$ and $\operatorname{St}(\mathcal{B})$ as $X_{1}$ and $X_{2}$. We form the sequences of subsets $A_{0}, A_{1}, \ldots$ and $B_{0}, B_{1}, \ldots$ of $X_{1}$ and $X_{2}$, noting that since the function $d$ is bounded, $\mathcal{A}_{0} \cap \mathcal{B}_{0}$ is trivial, and there is some largest integer $N$ such that $B_{N}$ is non-empty. Then the previous lemma applies to show that the charges $\mu_{0}$ and $\nu_{0}$ are automatically consistent and in fact have a common extension to $\mathcal{P}(R(\mathcal{A}, \mathcal{B}))$. The restriction of this charge to the clopen subsets of $R(\mathcal{A}, \mathcal{B})$ yields a $G$-valued extension charge on $\mathcal{A} \vee \mathcal{B}$.

N.B. It is worth noting that we have proved something a little stronger than the statement of the theorem: in fact, we have shown that under this hypothesis on $d$, any extensions of the original charges to the power set algebras of the corresponding Stone spaces have a common extension; in particular, they are automatically consistent! 
We present a special case where $d$ is bounded.

3.3. Corollary. Suppose that $\mathcal{A} \cap \mathcal{B}$ is trivial and that $R(\mathcal{A}, \mathcal{B})$ is open in $\operatorname{St}(\mathcal{A}) \times \operatorname{St}(\mathcal{B})$. Then the function $d$ is bounded, and so $\mathcal{A}$ and $\mathcal{B}$ always admit common extensions, i.e. $H(\mathcal{A}, \mathcal{B})$ is free.

Proof. Since $R(\mathcal{A}, \mathcal{B})$ is compact, we see that it is really a finite union of clopen rectangles $C_{i} \times D_{j}$. The fields $\mathcal{C}$ and $\mathcal{D}$ generated by these two respective collections of sets $C_{i}$ and $D_{j}$ are finite and so are atomic with atoms $A_{1}, \ldots, A_{m}$ and $B_{1}, \ldots, B_{n}$. It is easy to see that $d \leq m+n$.

We summarize the results obtained so far by means of the following diagram:

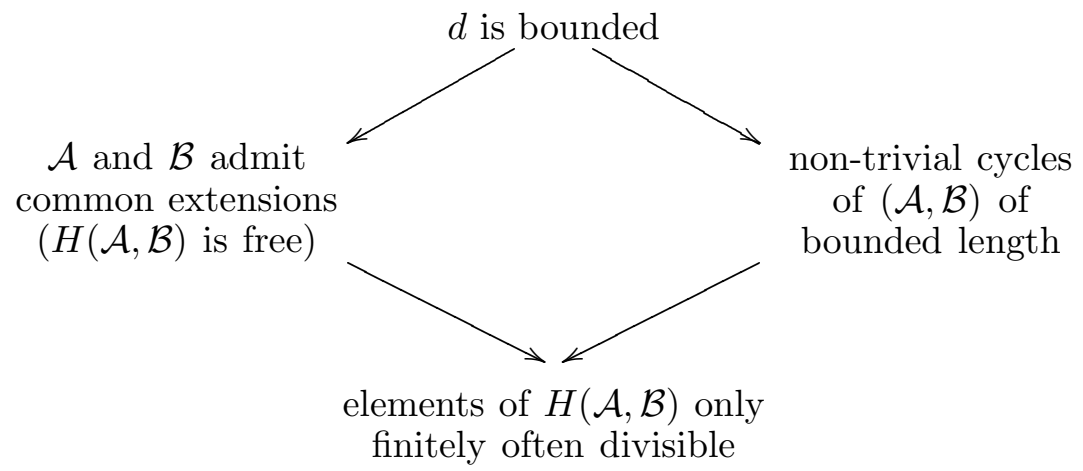

The implication upper left in the diagram cannot be reversed, as shown by the following construction suggested by L. C. Robertson.

3.4. Example. Define a subset of the plane as follows:

$$
R=\bigcup_{n=1}^{\infty}\left\{\left(1-\frac{1}{2^{n+1}}, 1-\frac{1}{2^{n}}\right),\left(1-\frac{1}{2^{n}}, 1-\frac{1}{2^{n}}\right)\right\} \cup\left\{\left(\frac{1}{2}, 1\right),(1,1)\right\} .
$$

Define $X_{1}=X_{2}=\left\{1-1 / 2^{n}: n \geq 1\right\} \cup\{1\}$. Then the $X_{i}$ and $R$ are compact, zero-dimensional spaces with $R \subseteq X_{1} \times X_{2}$. Define fields

$$
\begin{aligned}
& \mathcal{A}=\left\{\left(C \times X_{2}\right) \cap R: C \text { clopen subset of } X_{1}\right\}, \\
& \mathcal{B}=\left\{\left(X_{1} \times C\right) \cap R: C \text { clopen subset of } X_{2}\right\} .
\end{aligned}
$$

Then $\mathcal{A}$ and $\mathcal{B}$ are fields of subsets of $R$ with $\mathcal{A} \cap \mathcal{B}=\{\emptyset, R\}$. Also, $X_{1} \cong$ $\operatorname{St}(\mathcal{A})$ and $X_{2} \cong \operatorname{St}(\mathcal{B})$; under this identification, $R=R(\mathcal{A}, \mathcal{B})$. It is easy to see that $d$ is not bounded, although it never takes the value $\infty$. However, the group $H(\mathcal{A}, \mathcal{B})$ is free. In fact, $H(\mathcal{A}, \mathcal{B}) \cong \mathbb{Z}$, and the indicator of any point of $R$ other than $(1,1)$ serves as a generator.

3.5. Question. Can the other three implications in the diagram be reversed? 
4. Independent and locally independent fields. The notion of independence of fields of sets was apparently introduced by Marczewski, who proved that consistent, non-negative $\mathbb{R}$-valued charges on independent fields always had a non-negative common extension [10].

There is a weaker notion of "almost independence", used by Lipecki [9], who investigated bounded extensions of bounded charges. (Compare [2].) Independence was applied to group-valued charges in [3], where a complicated algebraic proof was used to show that $H(\mathcal{A}, \mathcal{B})$ is free whenever $\mathcal{A}$ and $\mathcal{B}$ are independent. In this section, a simpler proof is given using the distance function $d$ : the method applies also to the case of almost independent fields.

Let $\mathcal{A}$ and $\mathcal{B}$ be fields of subsets of a non-empty set $X$. Say that $\mathcal{A}$ is locally independent of $\mathcal{B}$ if for all partitions of $X=A_{1} \cup \ldots \cup A_{m}=$ $B_{1} \cup \ldots \cup B_{n}$ into $\mathcal{A}$ - and $\mathcal{B}$-sets, there exists some $i_{0}$ so that $A_{i} \cap B_{i_{0}} \neq \emptyset$ for $i=1, \ldots, m$.

Note that the definition is asymmetric in $\mathcal{A}$ and $\mathcal{B}$ and that $\mathcal{A}$ and $\mathcal{B}$ are almost independent (see Lipecki [9]) if and only if $\mathcal{A}$ is locally independent of $\mathcal{B}$ and also $\mathcal{B}$ is locally independent of $\mathcal{A}$.

The proof of the following result was suggested to the second author by A. Molitor.

4.1. Theorem. Let $\mathcal{A}$ and $\mathcal{B}$ be fields of subsets of a non-empty set $X$ with $\mathcal{A}$ locally independent of $\mathcal{B}$. For every partition of $X=B_{1} \cup \ldots \cup B_{n}$ into $\mathcal{B}$-sets, there is some $i_{0}$ such that $A \cap B_{i_{0}} \neq \emptyset$ for every non-empty $A \in \mathcal{A}$.

P r o of. Let $\operatorname{St}(\mathcal{B})$ be the Stone space for the algebra $\mathcal{B}$ and let $\varphi: \mathcal{B} \rightarrow \mathcal{C}$ be the canonical isomorphism between $\mathcal{B}$ and the algebra $\mathcal{C}$ of clopen subsets of $\operatorname{St}(\mathcal{B})$. For each pair $\pi=\left(A_{1}, \ldots, A_{m}, B_{1}, \ldots, B_{n}\right)$ of partitions of $X=$ $A_{1} \cup \ldots \cup A_{m}=B_{1} \cup \ldots \cup B_{n}$ into sets $A_{i} \in \mathcal{A}$ and $B_{i} \in \mathcal{B}$, define

$$
K(\pi)=\bigcup\left\{\varphi\left(B_{i}\right): B_{i} \cap A_{j} \neq \emptyset \text { for all } j=1, \ldots, m\right\} .
$$

It follows from local independence that each $K(\pi)$ is non-empty. The family of all sets $K(\pi)$ has the finite intersection property: if $\pi$ and $\pi^{\prime}$ are pairs of partitions, we let $\pi^{\prime \prime}$ be the pair of common refinements of the partitions in $\pi$ and $\pi^{\prime}$; then $K\left(\pi^{\prime \prime}\right) \subseteq K(\pi) \bigcap K\left(\pi^{\prime}\right)$. Since each $K(\pi)$ is compact, there is some point $s \in \bigcap_{\pi} K(\pi)$. The point $s$ represents an ultrafilter $\mathcal{U} \subseteq \mathcal{B}$.

Given a partition $X=B_{1} \cup \ldots \cup B_{m}$ with $B_{i} \in \mathcal{B}$, we find $i_{0}$ so that $B_{i_{0}} \in \mathcal{U}$. Given a non-empty $A \in \mathcal{A}$, we consider the pair of partitions $\pi=\left(A, X-A, B_{1}, \ldots, B_{n}\right)$. (We may assume that $A \neq X$.) Now $s \in K(\pi)$ and $s \in \varphi\left(B_{i_{0}}\right)$, so that $B_{i_{0}} \cap A \neq \emptyset$, as required.

Note. Considering the representation of $\mathcal{A} \vee \mathcal{B}$ as the clopen algebra on $R(\mathcal{A}, \mathcal{B})$, we have proved the existence of some $s \in \operatorname{St}(\mathcal{B})$ such that $\operatorname{St}(\mathcal{A}) \times\{s\} \subseteq R(\mathcal{A}, \mathcal{B})$. 
Two fields $\mathcal{A}$ and $\mathcal{B}$ on $X$ are said to be independent if $A \cap B \neq \emptyset$ whenever $A \in \mathcal{A}$ and $B \in \mathcal{B}$ are non-empty. Clearly, independence implies local independence.

4.2. Theorem. Let $\mathcal{A}$ and $\mathcal{B}$ be fields of subsets of a set $X$ with $\mathcal{A}$ locally independent of $\mathcal{B}$ (e.g., if $\mathcal{A}$ and $\mathcal{B}$ are independent). Then $H(\mathcal{A}, \mathcal{B})$ is free.

Pro of. Under this hypothesis, it is easy to use Theorem 4.1 and verify that $d \leq 3$. Then apply Theorem 3.2.

5. Compact metric spaces. In this section, we apply the method outlined in the power set Lemma 3.1 to a special case where $\mathcal{A}$ and $\mathcal{B}$ arise as subfields of Borel subsets of a compact metric space.

5.1. Theorem. Let $f: K \rightarrow \mathbb{R}$ and $g: K \rightarrow \mathbb{R}$ be continuous functions on a compact metric space $K$. Let $\mathcal{A}$ and $\mathcal{B}$ be the spectral fields generated by $f$ and $g$ as follows:

$$
\begin{aligned}
& \mathcal{A}=\left\{f^{-1}(C): C \text { a Borel subset of } \mathbb{R}\right\}, \\
& \mathcal{B}=\left\{g^{-1}(C): C \text { a Borel subset of } \mathbb{R}\right\} .
\end{aligned}
$$

Suppose that $\mathcal{A} \cap \mathcal{B}=\{\emptyset, K\}$. The following conditions are equivalent:

(i) There is some integer $M$ such that every cycle for $(\mathcal{A}, \mathcal{B})$ is of length less than $M$.

(ii) For any group $G$, any two consistent $G$-valued charges on $\mathcal{A}$ and $\mathcal{B}$ have a common extension to a $G$-valued charge on $\mathcal{A} \vee \mathcal{B}$, i.e. $H(\mathcal{A}, \mathcal{B})$ is free.

Proof. We very closely imitate the proof of Lemma 3.1. Define $X_{1}=$ range $(f)$ and $X_{2}=\operatorname{range}(g)$ and the function $\Psi: K \rightarrow X_{1} \times X_{2}$ by $\Psi(x)=$ $(f(x), g(x))$. Put $X=\operatorname{range}(\Psi)$ and define fields $\mathcal{A}_{0}$ and $\mathcal{B}_{0}$ on $X$ by

$$
\begin{aligned}
& \mathcal{A}_{0}=\left\{\left(A \times X_{2}\right) \cap X: A \text { a Borel subset of } X_{1}\right\}, \\
& \mathcal{B}_{0}=\left\{\left(X_{1} \times B\right) \cap X: B \text { a Borel subset of } X_{2}\right\} .
\end{aligned}
$$

Since $\mathcal{A} \cap \mathcal{B}$ is trivial, so is $\mathcal{A}_{0} \cap \mathcal{B}_{0}$.

(i) $\Rightarrow$ (ii). Just as in the proof of Lemma 3.1 , we take $A_{0}$ to be an arbitrary singleton subset of $X_{1}$ and define the sets $R_{0}, R_{1}, \ldots, T_{0}, T_{1}, \ldots, A_{1}, \ldots$, and $B_{0}, B_{1}, \ldots$ Since $X$ is compact, so are $R_{i}$ and $A_{0} \cup \ldots \cup A_{i}$ and $B_{0} \cup \ldots \cup B_{i}$ for each $i$. Thus, each $A_{i}$ [resp. $B_{i}$ ] is a Borel subset of $X_{1}$ [resp. $X_{2}$ ]. As in the proof of 3.1, $A_{i}=B_{i}=\emptyset$ for $i \geq M$. As in the proof of 3.1, construct graphs $G_{i} \subseteq T_{i}$.

Suppose now that $\mu$ and $\nu$ are consistent $G$-valued charges on $\mathcal{A}$ and $\mathcal{B}$. Define $\mu_{0}$ and $\nu_{0}$ on $\mathcal{A}_{0}$ and $\mathcal{B}_{0}$ by $\mu_{0}\left(\left(A \times X_{2}\right) \cap X\right)=\mu\left(f^{-1}(A)\right)$ and similarly for $B \subseteq X_{2}$. As in 3.1, we can view $\mu_{0}$ and $\nu_{0}$ as charges defined on (the Borel subsets of) $X_{1}$ and $X_{2}$. Using Lemma 1.1, these charges $\mu_{0}$ and 
$\nu_{0}$ may be extended to $\mathcal{P}\left(X_{1}\right)$ and $\mathcal{P}\left(X_{2}\right)$. This we do and define the charges $p_{i}$ and $p$ by the same formulae as in the proof of 3.1: here, $p$ is defined on $\mathcal{P}(X)$. Let $\tau$ be any charge on $\mathcal{A} \vee \mathcal{B}$ such that $\tau\left(\Psi^{-1}(C)\right)=p(C)$ for all $C \subseteq X$. This $\tau$ extends $\mu$ and $\nu$.

N ot e. There is, as with the proof of Theorem 3.2, great freedom here in the extension of $\mu_{0}$ and $\nu_{0}$ to $\mathcal{P}\left(X_{1}\right)$ and $\mathcal{P}\left(X_{2}\right)$-any two such extensions are automatically consistent.

(ii) $\Rightarrow$ (i). Once again, we follow the proof of Lemma 3.1. We find that $X_{1}=\bigcup A_{i}$ and $X_{2}=\bigcup B_{i}$ and that there is some $M$ such that $A_{i}=B_{i}=\emptyset$ for $i \geq M$. Then $M+1$ is an upper bound on the length of cycles for $(\mathcal{A}, \mathcal{B})$.

5.2. Question. Is Theorem 6.1 true when the space $K$ is not assumed compact (perhaps only complete and separable) or if $f$ and $g$ are not continuous, but only Borel measurable? These conditions were used (in both parts of proof) to show that the $A_{i}$ and $B_{i}$ are Borel.

6. The class of groups $H(\mathcal{A}, \mathcal{B})$. In this section, we consider the question of which (torsion-free) groups can arise as $H(\mathcal{A}, \mathcal{B})$ for appropriately chosen $\mathcal{A}$ and $\mathcal{B}$. We begin by showing that every subgroup of $\mathbb{Q}$ can be realized in this way.

Every subgroup of $H$ of $\mathbb{Q}$ is a direct limit of copies of $\mathbb{Z}$, i.e. there is a sequence $\left(n_{2}, n_{3}, \ldots\right)$ of integers $n_{k}>1$ and a system of groups and homomorphisms

$$
H_{k} \stackrel{\varphi_{k}}{\longrightarrow} H_{k+1}, \quad H_{k} \stackrel{\psi_{k}}{\longrightarrow} H \quad(k \geq 1)
$$

with $H_{k} \cong H_{k+1} \cong \mathbb{Z}, \varphi_{k}(m)=n_{k+1} m$, and $\psi_{k+1} \circ \varphi_{k}=\psi_{k}$ such that $\psi_{k}$ is one-one and $H=\bigcup \psi_{k}\left(H_{k}\right)$. Let $p_{1}<p_{2}<\ldots$ be the sequence of all prime numbers. Then given a sequence $\left(n_{1}, n_{2}, \ldots\right)$ we define $\chi_{r}=\sup \{n$ : $p_{r}^{n} \mid \prod_{i=1}^{k} n_{i}$ for all large $\left.k\right\}$. Then $\chi=\left(\chi_{r}\right)_{r \in \omega}$ is the characteristic of the sequence $\left(n_{i}\right)$. For characteristic and the related notion of type, see [7; Vol. II, p. 108]. Two such groups $H$ and $H^{\prime}$ are isomorphic if and only if their types described above are equal (see [7; Vol. II, p. 110, Theorem 85.1]).

6.1. Theorem. For every subgroup $H$ of $\mathbb{Q}$, there are a set $X$ and fields $\mathcal{A}$ and $\mathcal{B}$ of subsets of $X$ such that $\mathcal{A} \cap \mathcal{B}=\{\emptyset, X\}$ and $H(\mathcal{A}, \mathcal{B}) \cong H$.

P r o of. We consider $H$ as a direct limit of copies of $\mathbb{Z}$ as above with corresponding sequence $\left(n_{2}, n_{3}, \ldots\right)$. Put $n_{1}=1$. Define sets $X_{k}=\{0,1,2, \ldots$, $\left.n_{k}-1\right\}$ for $k=1,2, \ldots$ and put $P_{0}=X_{1} \times X_{2} \times \ldots$ For each $x \in P_{0}$, we write $x=(x(1), x(2), \ldots)$. Define

$$
\begin{array}{r}
E=\left\{x \in P_{0}: \text { either }(\exists N)(x(k)=0 \text { for all } k \geq N)\right. \\
\text { or } \left.(\exists N)\left(x(k)=n_{k}-1 \text { for all } k \geq N\right)\right\}
\end{array}
$$


and put $P=P_{0} \backslash E$. For each $m$, let $\mathcal{C}_{m}$ be the field on $P$ generated by all $m$-dimensional rectangles of the form $R=\left\{x \in P: x(1)=k_{1}, \ldots, x(m)\right.$ $\left.=k_{m}\right\}$. Then put $\mathcal{C}=\bigcup \mathcal{C}_{m}$.

We will define $X$ as a subset of $P \times P$ by means of a partition of $X=$ $D \cup F_{1} \cup F_{2} \cup \ldots$ First, we set $D=\{(x, x): x \in P\}$, the diagonal in $P \times P$. Then we put

$$
\begin{array}{r}
F_{k}=\left\{(x, y) \in P \times P: x(i)=0 \text { and } y(i)=n_{i}-1 \text { for all } i<k ;\right. \\
x(k)=y(k)+1 ; x(j)=y(j) \text { for all } j>k\} .
\end{array}
$$

It is not hard to verify that $F=\bigcup F_{k}$ is the (graph of) a one-one $\mathcal{C}$ measurable function from $P$ onto $P$. This completes the definition of $X$.

Let $\pi_{1}: P \times P \rightarrow P$ [resp. $\pi_{2}: P \times P \rightarrow P$ ] be projection to the first [resp. second] factor of $P \times P$. Define

$$
\mathcal{A}_{k}=\left\{X \cap \pi_{1}^{-1}(C): C \in \mathcal{C}_{k}\right\}, \quad \mathcal{B}_{k}=\left\{X \cap \pi_{2}^{-1}(C): C \in \mathcal{C}_{k}\right\} .
$$

These and $\mathcal{A}=\cup \mathcal{A}_{k}, \mathcal{B}=\bigcup \mathcal{B}_{k}$ are fields of subsets of $X$. For each $k=$ $1,2, \ldots$, define $u_{k}: P \rightarrow \mathbb{Z}$ by the formula

$$
u_{k}(x)=x(1)+x(2) n_{1}+x(3) n_{1} n_{2}+\ldots+x(k) n_{1} \ldots n_{k-1} .
$$

Then the atoms of the finite field $\mathcal{A}_{k}$ are given by

$$
A_{i}^{k}=\left\{(x, y) \in X: u_{k}(x)=i\right\}, \quad i=0,1, \ldots, n_{1} \ldots n_{k}-1,
$$

and those of $\mathcal{B}_{k}$ by

$$
B_{i}^{k}=\left\{(x, y) \in X: u_{k}(y)=i\right\}, \quad i=0,1, \ldots, n_{1} \ldots n_{k}-1 .
$$

It is simple to check that $A_{i}^{k} \cap B_{j}^{k} \neq \emptyset$ exactly when $i=j$ or $i=j+1$ or both $i=0$ and $j=n_{1} \ldots n_{k}-1$.

It is easy to check that $H_{k}=H\left(\mathcal{A}_{k}, \mathcal{B}_{k}\right) \cong \mathbb{Z}$ with generator $h_{k}+S\left(\mathcal{A}_{k}\right)+$ $S\left(\mathcal{B}_{k}\right)$, where $h_{k}$ is the indicator function of the set $A_{n_{1} \ldots n_{k}-1}^{k} \cap B_{n_{1} \ldots n_{k}-1}^{k}$. We see that

$$
h_{k}(x, y)= \begin{cases}1 & \text { if } x=y \text { and } x(i)=n_{i}-1 \text { for all } i \leq k, \\ 0 & \text { otherwise. }\end{cases}
$$

Now define $e=I_{F}$. Since $D=\{(x, y): x(1)=y(1)\}$, we see that $D$ and hence also $F$ is $\mathcal{A}_{1} \vee \mathcal{B}_{1}$-measurable. We also define, for each $k \geq 1$, functions $f_{k}$ and $g_{k}$ on $X$ :

$$
\begin{aligned}
& f_{k}(x, y)=u_{k}(x), \\
& g_{k}(x, y)= \begin{cases}1 & \text { if } y(i)=n_{i}-1 \text { for all } i \leq k, \\
-u_{k}(y) & \text { otherwise. }\end{cases}
\end{aligned}
$$

From what has already been said, it is clear that $f_{k} \in S\left(\mathcal{A}_{k}\right)$ and $g_{k} \in S\left(\mathcal{B}_{k}\right)$.

Claim. For each $k \geq 1$, we have the equation $e=f_{k}+g_{k}-n_{1} \ldots n_{k} h_{k}$. 
Proof of claim. We hold $k$ and $p \in X$ fixed and distinguish four cases.

Case 1. $p=(x, x)$ and $h_{k}(p)=0$. In this case $e(p)=0, f_{k}(p)=u_{k}(x)$ and $g_{k}(p)=-u_{k}(x)$, so that the equation follows easily.

Case 2. $p=(x, x)$ and $h_{k}(p)=1$. Then $e(p)=0$ and $g_{k}(p)=1$. Also,

$$
\begin{aligned}
f_{k}(p) & =u_{k}(x) \\
& =\left(n_{1}-1\right)+\left(n_{2}-1\right) n_{1}+\left(n_{3}-1\right) n_{1} n_{2}+\ldots+\left(n_{k}-1\right) n_{1} n_{2} \ldots n_{k-1} \\
& =n_{1} n_{2} \ldots n_{k}-1,
\end{aligned}
$$

so the equation is satisfied.

Case 3. $p=(x, y) \in F$ with $x(1)=x(2)=\ldots=x(k)=0$. Then it follows that $p \in F_{m}$ for some $m \geq k+1$, so that $y(i)=n_{k}-1$ for all $i \leq k$. Thus $f_{k}(p)=u_{k}(x)=0$ and $g_{k}(p)=1$. Also, $e(p)=1$ and $h_{k}(p)=0$, so the equation follows.

Case 4. $p=(x, y) \in F_{m}$ for some $m \leq k$. Then

$$
\begin{aligned}
f_{k}(p)= & u_{k}(x) \\
= & x(1)+x(2) n_{1}+\ldots+x(m-1) n_{1} n_{2} \ldots n_{m-2} \\
& +x(m) n_{1} \ldots n_{m-1}+x(m+1) n_{1} \ldots n_{m}+\ldots+x(k) n_{1} \ldots n_{k-1}, \\
g_{k}(p)= & -u_{k}(y) \\
= & -y(1)-y(2) n_{1}-\ldots-y(m-1) n_{1} \ldots n_{m-2} \\
& -y(m) n_{1} \ldots n_{m-1}-y(m+1) n_{1} \ldots n_{m}-\ldots-y(k) n_{1} \ldots n_{k-1} .
\end{aligned}
$$

Now $x(i)=0$ and $y(i)=n_{i}-1$ for all $i<m$; also, $x(m)=y(m)+1$; and $x(j)=y(j)$ for all $j>m$. It follows that

$$
\begin{aligned}
f_{k}(p) & +g_{k}(p) \\
& =n_{1} \ldots n_{m-1}-\left(n_{1}-1\right)-\left(n_{2}-1\right) n_{1}-\ldots-\left(n_{m-1}-1\right) n_{1} \ldots n_{m-2} \\
& =n_{1} \ldots n_{m-1}-\left(n_{1} \ldots n_{m-1}-1\right)=1 .
\end{aligned}
$$

Also, $e(p)=1$ and $h_{k}(p)=0$, and the claim is thus established.

We now define, for each $k \geq 1$, a function $\psi_{k}: H_{k} \rightarrow H(\mathcal{A}, \mathcal{B})$ by putting $\psi_{k}\left(h+S\left(\mathcal{A}_{k}\right)+S\left(\mathcal{B}_{k}\right)\right)=h+S(\mathcal{A})+S(\mathcal{B})$. Since $S\left(\mathcal{A}_{k}\right) \subseteq S(\mathcal{A})$ and $S\left(\mathcal{B}_{k}\right) \subseteq S(\mathcal{B})$, it follows that $\psi_{k}$ is a well-defined group homomorphism. In order to prove that $\psi_{k}$ is injective, it suffices to prove that $h_{k} \notin S(\mathcal{A})+S(\mathcal{B})$. This is sufficient for two reasons: the coset of $h_{k}$ generates $H_{k}$, and the subgroup $S(\mathcal{A})+S(\mathcal{B})$ is pure in $S(\mathcal{A} \vee \mathcal{B})$. But, again using the purity of $S(\mathcal{A})+S(\mathcal{B})$, we see from the equation $e=f_{k}+g_{k}-n_{1} \ldots n_{k} h_{k}$ that $h_{k} \in S(\mathcal{A})+S(\mathcal{B})$ if and only if $e \in S(\mathcal{A})+S(\mathcal{B})$. But then there is some $N$ 
such that $e \in S\left(\mathcal{A}_{N}\right)+S\left(\mathcal{B}_{N}\right)$. From the equation $e=f_{N}+g_{N}-n_{1} \ldots n_{N} h_{N}$, we see (purity again!) that $h_{N} \in S\left(\mathcal{A}_{N}\right)+S\left(\mathcal{B}_{N}\right)$, a contradiction. So $\psi_{k}$ is injective.

In fact, we have proved that, for each $k,(S(\mathcal{A})+S(\mathcal{B})) \cap S\left(\mathcal{A}_{k} \vee \mathcal{B}_{k}\right)=$ $S\left(\mathcal{A}_{k}\right)+S\left(\mathcal{B}_{k}\right)$.

Next, we define $\varphi_{k}: H_{k} \rightarrow H_{k+1}$ by putting $\varphi_{k}\left(h+S\left(\mathcal{A}_{k}\right)+S\left(\mathcal{B}_{k}\right)\right)=$ $h+S\left(\mathcal{A}_{k+1}\right)+S\left(\mathcal{B}_{k+1}\right)$. Then $\varphi_{k}$ is a well-defined homomorphism. From the equation of the claim, we may write

$$
f_{k}+g_{k}-n_{1} \ldots n_{k} h_{k}=e=f_{k+1}+g_{k+1}-n_{1} \ldots n_{k} n_{k+1} h_{k+1} .
$$

Thus $n_{1} \ldots n_{k+1} h_{k+1}-n_{1} \ldots n_{k} h_{k}=n_{1} \ldots n_{k}\left(n_{k+1} h_{k+1}-h_{k}\right)$ is an element of $S\left(\mathcal{A}_{k+1}\right)+S\left(\mathcal{B}_{k+1}\right)$ : by purity, so also is $n_{k+1} h_{k+1}-h_{k}$. Thus, considered as a mapping between cyclic groups $H_{k}$ and $H_{k+1}, \varphi_{k}$ is multiplication by $n_{k+1}$. The conditions $\psi_{k+1} \circ \varphi_{k}=\psi_{k}$ and $H(\mathcal{A}, \mathcal{B})=\bigcup \psi_{k}\left(H_{k}\right)$ are easy to see.

Thus, we have realized $H$ as $H(\mathcal{A}, \mathcal{B})$ for fields $\mathcal{A}$ and $\mathcal{B}$ on $X$, as required. The condition $\mathcal{A} \cap \mathcal{B}=\{\emptyset, X\}$ follows easily from the fact that $\mathcal{A}_{k} \cap \mathcal{B}_{k}=$ $\{\emptyset, X\}$ for each $k$.

Our next step is to show that the class of groups $H(\mathcal{A}, \mathcal{B})$ is closed under the taking of direct sums. Let $\left\{X_{i}: i \in I\right\}$ be a family of disjoint non-empty sets and let $\left\{\mathcal{A}_{i}: i \in I\right\}$ be a corresponding family of fields $\mathcal{A}_{i}$ on $X_{i}$. Put $X=\bigcup\left\{X_{i}: i \in I\right\}$ and define $\sum \mathcal{A}_{i}$ as the field comprising all sets $\bigcup\left\{A_{i}: i \in I\right\}$ such that $A_{i} \in \mathcal{A}_{i}$ for each $i$, and either

(i) for all but finitely many $i \in I$, we have $A_{i}=\emptyset$, or

(ii) for all but finitely many $i \in I, A_{i}=X_{i}$.

Then $\sum \mathcal{A}_{i}$ is the field generated by $\bigcup \mathcal{A}_{i}$. So $\left(\sum \mathcal{A}_{i}\right) \vee\left(\sum \mathcal{B}_{i}\right)=\sum\left(\mathcal{A}_{i} \vee \mathcal{B}_{i}\right)$.

6.2. Lemma. Let $X_{i}(i \in I)$ be a family of disjoint non-empty sets as above with fields $\mathcal{A}_{i}$ and $\mathcal{B}_{i}$ on $X_{i}$. Then

$$
\frac{S\left(\left(\sum \mathcal{A}_{i}\right) \vee\left(\sum \mathcal{B}_{i}\right)\right)}{S\left(\sum \mathcal{A}_{i}\right)+S\left(\sum \mathcal{B}_{i}\right)} \cong \bigoplus_{i \in I} \frac{S\left(\mathcal{A}_{i} \vee \mathcal{B}_{i}\right)}{S\left(\mathcal{A}_{i}\right)+S\left(\mathcal{B}_{i}\right)}
$$

Proof. We exhibit an isomorphism $\varphi$ from the right member of the isomorphism to the left. Let $\left(\bar{f}_{1}, \bar{f}_{2}, \ldots\right)$ be a sequence in the product, where each $f_{i} \in S\left(\mathcal{A}_{i} \vee \mathcal{B}_{i}\right)$ and $\bar{f}_{i}=f_{i}+S\left(\mathcal{A}_{i}\right)+S\left(\mathcal{B}_{i}\right)$. Put $\varphi\left(\bar{f}_{1}, \bar{f}_{2}, \ldots\right)=f$, where $f: X \rightarrow \mathbb{Z}\left(X=\bigcup X_{i}\right)$ is defined by $f(x)=f_{i}(x)$ for $x \in X_{i}$. It is easy to check that $\varphi$ is well defined and provides the desired isomorphism.

A consequence of this lemma is that all free groups are realizable as groups of the form $H(\mathcal{A}, \mathcal{B})$.

6.3. Question. Is there a torsion-free group not isomorphic to $H(\mathcal{A}, \mathcal{B})$ for any $\mathcal{A}$ and $\mathcal{B}$ ? 
7. Cotorsion theories and the problem of common extensions. If $R$ is a subgroup of $\mathbb{Q}$, then we can find a pair $\mathcal{A}_{R}, \mathcal{B}_{R}$ of fields on a set $X_{R}$ such that $\mathcal{A}_{R} \cap \mathcal{B}_{R}=\left\{\emptyset, X_{R}\right\}$ and $H\left(\mathcal{A}_{R}, \mathcal{B}_{R}\right)=S\left(\mathcal{A}_{R} \vee \mathcal{B}_{R}\right) /\left(S\left(\mathcal{A}_{R}\right)+\right.$ $\left.S\left(\mathcal{B}_{R}\right)\right) \cong R$ (Theorem 6.1).

We say that a group $G$ has the lifting property for $R$ if all consistent charges $\sigma_{\mathcal{A}}: \mathcal{A}_{R} \rightarrow G$ and $\sigma_{\mathcal{B}}: \mathcal{B}_{R} \rightarrow G$ can be extended to a common extension $\sigma: \mathcal{P}\left(X_{R}\right) \rightarrow G$. According to Lemma 1.2 this is equivalent to saying that

$$
\operatorname{Ext}(R, G)=0
$$

for a given rational group $R$ as above. Fortunately this question (and a little more) can be answered by some progress in Abelian groups from about a decade ago.

The groups $G$ in $(*)$ are collected in a class $\{R\}^{\perp}=\{G: \operatorname{Ext}(R, G)=0\}$ (cf. [12; p. 12] and [8]). This class $\{R\}^{\perp}$ is the so-called "cotorsion component" of a "cotorsion theory cogenerated by $R$ ". The other component is called the "cotorsion-free component" $F\{R\}$ of this cotorsion theory and is defined to be $F\{R\}=\left\{Y: \operatorname{Ext}(Y, G)=0\right.$ for all $\left.G \in\{R\}^{\perp}\right\}$. If $R=\mathbb{Q}$, then the groups in $\{\mathbb{Q}\}^{\perp}$ are called cotorsion groups, a notion due to D. K. Harrison. An easy calculation shows that $F\{\mathbb{Q}\}$ is the class of all torsion-free groups (cf. [7; Vol. 1, p. 282]). Hence "rational cotorsion theories", which have these pairs $\left(F\{R\},\{R\}^{\perp}\right)$, are a natural generalization of the classical cotorsion theory. This extension can be found in [12].

One of the main theorems in [12] is a characterization of the groups $G$ in $\{R\}^{\perp}$ or equivalently of our groups $G$ with $(*)$. In order to state this result, we recall some notation. If $R \subseteq \mathbb{Q}$, then we may assume that $1 \in R$ without loss of generality. In this case we can say that the characteristic $\chi(R)$ is the characteristic $\chi(1)$ of $1 \in R$ which is a sequence $\left(r_{p}\right)(p \in \mathbb{P}=$ all primes $)$, where $r_{p}$ is the largest positive integer $n$ such that $p^{n}$ divides 1 in $R$. If $n$ does not exist, we set $r_{p}=\infty$. Then we put $G_{p}^{R}=G / p^{r_{p}} G$ if $r_{p}<\infty$ and $G_{p}^{R}=\operatorname{Ext}\left(\mathbb{Z}\left(p^{\infty}\right), G\right)$ if $r_{p}=\infty$. The group $G_{p}^{R}$ is bounded in the first case and the $p$-component of the classical cotorsion completion of $G$ in the second case (cf. [7; Vol. 1, pp. 224, 248, 249]), hence $G_{p}^{R}$ is cotorsion. Products of cotorsion groups are cotorsion, and $\prod_{p \in \mathbb{P}} G_{p}^{R}$ will always be cotorsion. If $G_{R}$ denotes the subgroup $\bigcap_{p \in \mathbb{P}} p^{r_{p}} G$ of $G$, then [12, Theorem 3.5] gives the following characterization:

7.1. THEOREM. If $R$ is a rational group, then the following are equivalent:

(i) $G \in\{R\}^{\perp}$.

(ii) $G / G_{R} \cong \prod_{p \in \mathbb{P}} G_{p}^{R}$.

(iii) $G / G_{R}$ is cotorsion. 
Indication: [12; p. 21].

If $G \in\{R\}^{\perp}$ is countable, then $G / G_{R}$ is also countable. Then, by Theorem 7.1(ii), we see that there is some finite $E \subseteq \mathbb{P}$ such that $G / G_{R} \cong$ $\prod_{p \in E} G_{p}^{R}$. There are then two cases to analyze:

Case 1 . If $G_{R}=0$, then $G / G_{R} \cong G \subseteq \mathbb{Q}$ is cotorsion, so that either $G=0$ or $G \cong \mathbb{Q}$.

Case 2. If $G_{R} \neq 0$, then $G / G_{R}$ is at once torsion and cotorsion. A theorem of Harrison and Nunke [7; Vol. 1, p. 235, Cor. 54.4] then says that $G / G_{R}=B \oplus D$, with $B$ a bounded group and $D$ divisible. We now prove $D=0$ and a little more using the isomorphism $G / G_{R} \cong \prod_{p \in E} G_{p}^{R}$. Since $G$ is of rank $1, G / p^{r_{p}} G \cong \mathbb{Z} p^{k_{p}}$, with $k_{p} \leq r_{p}$ for $r_{p}<\infty$. If $r_{p}=\infty$, then $G_{p}^{R}=\operatorname{Ext}\left(\mathbb{Z}\left(p^{\infty}\right), G\right)$, and $\operatorname{rank}(G)=1$ implies that the rank of a $p$-basic subgroup of $G$ is 1 or 0 . If it is 1 , then $G_{p}^{R}=\mathcal{I}_{p}$ (p-adic integers) and if 0 , then $G_{p}^{R}=0$; see [7; Vol. 1, p. 224]. The first case is excluded by the isomorphism $G / G_{R} \cong \prod_{p \in E} G_{p}^{R}$ and the restriction $\left|G / G_{R}\right|<2^{\omega}$. Hence $G_{p}^{R}=\operatorname{Ext}\left(\mathbb{Z}\left(p^{\infty}\right), G\right)=0$. We derive $B=\bigoplus\left\{\mathbb{Z} p^{k_{p}}: p \in E, r_{p}<\infty\right\}$ and $D=0$. We find $G / G_{R} \cong \bigoplus\left\{\mathbb{Z} p^{k_{p}}: p \in E^{\prime}\right\}$ with $E^{\prime}=\{p \in \mathbb{P}$ : either $R$ or $G$ is not divisible by $p\}$ a finite set. Since $p^{k_{p}} \mid R$ and $r_{p} \leq k_{p}$, also $k=\prod_{p \in E^{\prime}} p^{r_{p}} \mid R$ and $1 \in R^{\prime}$, where the mapping $x \rightarrow x / k$ is an isomorphism of $R$ onto $R^{\prime} \subseteq \mathbb{Q}$. If $\chi\left(R^{\prime}\right)=\left(r_{p}^{\prime}\right)$, then $r_{p}^{\prime}=0$ for all $p \in E^{\prime}$. Hence $G_{p}^{R}=0$ for $p \in E^{\prime}$, and $G_{R^{\prime}}=G$. Using Theorem 7.1 again, we have

7.2. Corollary. If $R$ and $G$ are rational groups, then $G \in\{R\}^{\perp}$ if and only if $R \cong R^{\prime}$ for some group $R^{\prime}$ with $R \subseteq R^{\prime} \subseteq \mathbb{Q}$ and $G_{R^{\prime}} \cong G$.

The corollary replaces the inadequate remark on p. 22 in [12; after Theorem 3.5]. It says that we can recognize the isomorphism classes of rational groups from their cotorsion classes $\{R\}^{\perp}$. If we consider only those rational groups $R$ with idempotent types (i.e. subrings of $\mathbb{Q}$ ), then we can, e.g., choose $R=R^{\prime}$.

We order two torsion theories by the inclusion of their cotorsion component. Then it follows that the set $(\mathcal{C}, \subseteq)$ of all rational cotorsion theories (or equivalently all their cotorsion parts $\{R\}^{\perp}$ ) is order isomorphic to the set of all isomorphism classes of rational groups. The latter has been characterized to be order isomorphic to the set of types, which is well understood (cf. [7; Vol. 2, p. 110, Theorem 85.1]. In particular, $|\mathcal{C}|=2^{\aleph_{0}}$. We will sumarize a special case applied to our lifting problem.

7.3. Corollary. There are pairs $\left(\mathcal{A}_{i}, \mathcal{B}_{i}\right)$ of fields on sets $X_{i}$ and groups $G_{i}$ for $i<2^{\aleph_{0}}$ such that $G_{j}$ has the lifting property for the fields $\left(\mathcal{A}_{i}, \mathcal{B}_{i}\right)$ if and only if $i=j$. 
This tells us that the lifting problem is at least as complex as the theory of types or equivalently the cotorsion theory of rational groups. Our result (6.1) can also be supplied for more general cotorsion theories [12]. If $(\mathcal{X}, \mathcal{Y})$ is a pair of classes of abelian groups, we say that $\mathcal{X} \perp \mathcal{Y}$ if and only if $\operatorname{Ext}(X, Y)=0$ for all $X \in \mathcal{X}$ and $Y \in \mathcal{Y}$. Generalizing $\{R\}^{\perp}$, we set $\mathcal{X}^{\perp}=\{Y: \mathcal{X} \perp Y\}$ and ${ }^{\perp} \mathcal{X}=\{Y: Y \perp \mathcal{X}\}$ (cf. [8]). We replaced $\{Y\}$ by $Y$ for simplicity. A pair $(\mathcal{X}, \mathcal{Y})$ is a cotorsion theory if the following holds:

(1) $\mathcal{X} \perp \mathcal{Y}$

(2) maximality of $\mathcal{Y}: \mathcal{X} \perp Y$ implies $Y \in \mathcal{Y}$;

(3) maximality of $\mathcal{X}: X \perp \mathcal{Y}$ implies $X \in \mathcal{X}$.

It follows that $\mathcal{X}^{\perp}=\mathcal{Y}$ and ${ }^{\perp} \mathcal{Y}=\mathcal{X}$, hence ${ }^{\perp}\left(\mathcal{X}^{\perp}\right)=\mathcal{X}$ and $\left({ }^{\perp} \mathcal{Y}\right)^{\perp}=\mathcal{Y}$. We have seen that the classes $\mathcal{X}, \mathcal{Y}$ are closed under the closure operations $F \mathcal{A}={ }^{\perp}\left(\mathcal{A}^{\perp}\right)$ and $C \mathcal{A}=\left({ }^{\perp} \mathcal{A}\right)^{\perp}$ respectively, and any cotorsion theory can be viewed as $\left(F \mathcal{X}, \mathcal{X}^{\perp}\right)($ cogenerated by $\mathcal{X})$ or as $\left({ }^{\perp} \mathcal{X}, C \mathcal{X}\right)$ generated by $\mathcal{X}$. Then $C \mathcal{X}$ is called the cotorsion closure of $\mathcal{X}$ and $F \mathcal{X}$ is the cotorsion-free closure of $\mathcal{X}$. This is compatible with the classical cotorsion theory (torsionfree, cotorsion $)=\left(F \mathbb{Q}, \mathbb{Q}^{\perp}\right)$.

Three "almost classical" cotorsion theories $\left(F \mathcal{X}, \mathcal{X}^{\perp}\right)$ are of special interest in group theory:

(a) the classical cotorsion theory, where $\mathcal{X}=\{\mathbb{Q}\}$;

(b) local cotorsion theory with $\mathcal{X}=\left\{\mathbb{Z}^{p}=\left\{n / p^{m}: n, m \in \mathbb{Z}\right\}: p\right.$ any prime\}; and

(c) $\mathcal{X}=\left\{\mathbb{Z}_{(p)}\right.$ : the localizations at any primes $\left.p\right\}$, called quasi-cotorsion theory.

It follows that cotorsion $\Rightarrow$ quasi-cotorsion $\Rightarrow$ local cotorsion (cf. $[12$; p. 27]). A reduced group $K$ in the (non-splitting) exact sequence $0 \rightarrow K \rightarrow$ $\prod_{p} \mathcal{I}_{p} \rightarrow \bigoplus \mathbb{Q} \rightarrow 0$ and $G=\bigoplus \mathcal{I}_{p}$ show that the mentioned cotorsion classes are a proper chain.

From (5.1) and the above theory we obtain a pair of fields $(\mathcal{A}, \mathcal{B})$ on $X$ with $H(\mathcal{A}, \mathcal{B})=\mathbb{Q}$ having consistent $G$-charges $\left(\sigma^{1}, \sigma^{2}\right)$ which do not lift to $\mathcal{P}(X)$. However, there is a pair of fields $\mathcal{A}_{q}, \mathcal{B}_{q}$ on $X_{q}$, e.g. $\mathcal{A}_{q}=\bigoplus_{p} \mathcal{A}_{p}$, $\mathcal{A}_{q}=\bigoplus_{p} \mathcal{A}_{p}$ with $H\left(\mathcal{A}_{p}, \mathcal{B}_{p}\right)=\mathbb{Z}_{(p)}$ with the lifting property into $G$ but not into $K$.

This strengthens a recent result in [2] and [11], where $G$-charges in (1) come from all pairs of fields. We also can use only one pair of fields $(\mathcal{A}, \mathcal{B})$ with $H(\mathcal{A}, \mathcal{B})=\mathbb{Q}$ to find out that $G$ must be cotorsion. We close with the question whether the class of groups $H(\mathcal{A}, \mathcal{B})$ is closed also under taking direct products. 


\section{References}

[1] A. Basile and K. P. S. Bhaskara Rao, Common extensions of group-valued charges, Boll. Un. Mat. Ital. 7 (5-A) (1991), 157-162.

[2] A. Basile, K. P. S. Bhaskara Rao and R. M. Shortt, Bounded common extensions of bounded charges, Proc. Amer. Math. Soc. 121 (1994), 137-143.

[3] K. P. S. Bhaskara Rao and R. M. Shortt, Common extensions for homomorphisms and group-valued charges, Rend. Circ. Mat. Palermo (2) Suppl. 28 (1992), 125-140.

[4] - - - Group-valued charges: common extensions and the finite Chinese remainder property, Proc. Amer. Math. Soc. 113 (1991), 965-972.

[5] T. Carlson and K. Prikry, Ranges of signed measures, Period. Math. Hungar. 13 (1982), 151-155.

[6] S. E. Dickson, A torsion theory for abelian categories, Trans. Amer. Math. Soc. 121 (1966), 223-235.

[7] L. Fuchs, Infinite Abelian Groups, Vols. I and II, Academic Press, New York, 1970 \& 1973.

[8] R. Göbel and R. Prelle, Solution of two problems on cotorsion abelian groups, Arch. Math. (Basel) 31 (1978), 423-431.

[9] Z. Lipecki, On common extensions of two quasi-measures, Czechoslovak Math. J. 36 (1986), 489-494.

[10] E. Marczewski, Measures in almost independent fields, Fund. Math. 38 (1951), $217-229$.

[11] K. M. Rangaswamy and J. D. Reid, Common extensions of finitely additive measures and a characterization of cotorsion Abelian groups, in: Proc. Curacao, Abelian Groups, Marcel Dekker, New York, 1993, 231-238.

[12] L. Salce, Cotorsion theories for abelian groups, Symposia Math. 23 (1979), 11-32.

FACHBEREICH 6-MATHEMATIK

UNIVERSITÄT GHS

45117 ESSEN, GERMANY
DEPARTMENT OF MATHEMATICS

WESLEYAN UNIVERSITY

MIDDLETOWN, CONNECTICUT 06459-0128

Received 10 March 1993;

in revised form 16 November 1993 and 28 March 1994 